\title{
An Adaptive Vision-based Sensor for Underwater Line Detection Employing Shape and Color Image Segmentation
}

\author{
Mario A. Jordán \\ Instituto Argentino de Oceanografía (IADO-CONICET) and Dto. de Ing. Elec. y de Comp. - Univ. Nac. del Sur (DIEC- \\ UNS), Bahía Blanca, Argentina \\ Email: mjordan@criba.edu.ar \\ Emanuel Trabes \\ Instituto Argentino de Oceanografía (IADO-CONICET), Bahía Blanca, Argentina \\ Email: etrabes@iado-conicet.gov.ar
}

\begin{abstract}
This work aims the design and implementation of an adaptive vision-based sensor for detecting a pipe on underwater scenes in real time. The motivation is focused to future applications of vision servo control in underwater vehicles. The approach employs color and shape image segmentation together with an adjust mechanism that aims continuously in time to reach the best setup of a parameter set of the color image segmentation. The sensor performs very well even in the case of large and rapid changes in the scene illumination. On the basis of many experiments carried out in real scenes and the comparison with similar algorithms in the state-of-the-art field on the same application, the approach gets a better positioning with respect to related results above all in the case of extremely changing and poor luminance conditions. As drawback, the required computation time to achieve optimal values for the first time (auto-tuning phase) may be large; contrary to the adaptive ongoing process, in where the optimization is much more agile.
\end{abstract}

Index Terms - underwater pipeline detection, vision-based sensor, HSV model, optimal parameter adjustments, changing illumination levels, adaptive sensor

\section{INTRODUCTION}

Vision-based systems for underwater navigation currently employ optic or acoustic devices which provides a video or an intermittent image for own localization and rate estimation with respect to the surrounding environment (Jordán et al., 2010). They basically extract visual features of the seafloor by image segmentation, searching for chromatic and topological characteristics like colors, texture, shapes and contours.

Image segmentation is a widely studied area with hundreds of different algorithms (Law et al., 2004). The existing techniques to perform image segmentation are quite numerous, containing basic procedures like thresholding, clustering, data compression, histogram,

Manuscript received September 17, 2014; revised December 26, 2014. edge detection, region-growing, spliting-and-merging, among the most referenced (Shapiro and Stockman, 2001; Szeliski, R., 2010).

An important application in the area of underwater navigation is the pipeline detection and path tracking. When processing videos in real time for visual servoing, the main requirement is the reliability of the identified line position which is feed back to the controller in order to generate proper course corrections. Here, eventual changes in the scene illumination may alter drastically the performance of the vision-based sensor. Many of the problems associated with the performance drop are, to a greater or lesser extent, the consequence of the inability of an existing technique of finding the useful information from a vast amounts of messy and noisy data available, where most of which are non-informative. Even when there exists useful techniques for automatic setup of parameters of the image segmentation (Cheng et al., 2001; Harrabi and Ben, 2011), the sensor will fail if there are not a sufficient number of additional (sometimes redundant) image attributes for the segmentation.

While most edge detection techniques have been developed for grayscale images, color images can provide additional information, for instance, noticeable edges between iso-luminant colors are useful cues but fail to be detected by grayscale edge operators (Szeliski, R., 2010). In (Sattar and Dudek, 2006) the success rate of three different and widely used color-based trackers were comparatively established, namely color blob segmentation, histogram matching and mean-shift tracking. It was found that algorithms based on color blob segmentation, work best in most of the cases, in either well illuminated, poorly illuminated, or noisy submarine scenes.

These alternatives are taken into account in order to introduce modifications in the color image segmentation to design an algorithm with ad-hoc adaptive features under unknown, unfavorable and changing luminance conditions. 
Particularly, this paper deals with the image segmentation from a moving video camera directed at a subaquatic scene with a pipe lying on the submarine floor. The work is aimed at developing a detection method of the pipe under changing luminance conditions. The method attempts to reach an optimal setup by online tuning of specific parameters of the color image segmentation. It is claimed by the authors that these parameters have a significant influence on the success of the detection. The formalism for the presented technique is framed as a maximization problem. To this end a concave objective function in the space of these influential parameters is constructed for each processed frame and an adjust mechanism for them is developed to achieve a maximum. Finally the performance of the proposed approach is tested in the auto-tuning phase starting from unknown luminance conditions as well as in the adaptation phase over the time. Comparative results of the presented adaptive approach with a technique that has a suitable but constant set of segmentation parameters, are illustrated in a case study.

\section{REAl Subaquatic ScEnE}

We start the description of an underwater scene and the object type to be identified and tracked. In Fig. 1 the typical silhouette of a duct (pipe or cable) on the seafloor is illustrated. We will say that the physical pipeline is identified at a time point when we can establish a tangent line with slant $\alpha$ and distance $x_{L}$ from a reference point (pivot point) to the vertical axis of the frame (Jordan et al, 2010). Clearly, the estimation of $\alpha$ and $x_{L}$ takes place on the focal plane under a metrics of vision and not in a physical space with Euclidean metrics.

Subaquatic scenarios usually mask the detection of the true color composition of the scene. Thus, objects in it look an almost monochromatic aspect, generally of a strong bluish tonality. Under low-level-illuminated scenes, objects therein will be losing brightness. Even though the dynamic range of luminosity can be increased if highly sensitive cameras and artificial illuminators are employed, the presence of turbidity in water will cause blurred images. Clearly, color information from the pipeline and its surroundings may be scarce to pattern recognition. Even though blob image segmentation can offer good results under low-level illuminated images (Sattar and Dudek, 2006), the success of the technique basically depends on appropriate settings of the thresholds.

\section{PRELIMINARY FORMALISM}

In formal mathematical terms, let $F$ be a set of connected pixels and a segmentation of $F$ be a partition of connected subsets $S_{j}$ with $F=\cup S_{i}$ and $S_{i} \cap S_{j}=\Phi$. Then let $P$ be a predicate of homogeneity be a characteristic satisfied by $S_{j} \in F$ so that

$$
P\left(S_{i}\right)=\text { true for all } S_{i}
$$

$P\left(S_{i} \cup S_{j}\right)=$ false for all $S_{j}$ be a neigbor of $S_{i}$
The predicate may mean any condition of membership for $S_{i}$, for instance, membership to a hue value interval, to a histogram for a color model channel, or to an image pattern. The predicates we are interested are related to color and profile of the line in the background.

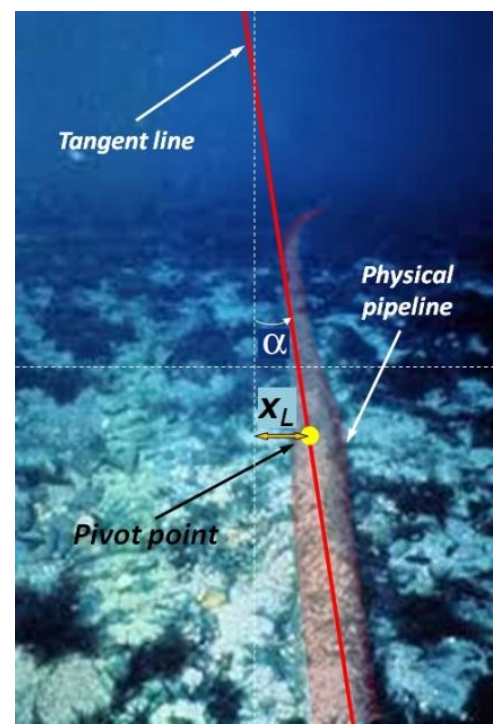

Figure 1. Subaquatic pipeline and identification of the AUV relative position for line tracking

\section{A. Color Predicate}

Let us consider here a HSV model with the hue, the saturation and the value channels.

We define the color predicate $P_{c}($.$) as the color$ attribute of a set $S_{\text {patch }}$ referred to as patch that holds

$$
\begin{gathered}
P_{c}\left(S_{\text {patch }}\right) \triangleq \text { true if for all } P_{i j} \in S_{\text {patch }} \text { we have } \\
\qquad H\left(p_{i j}\right) \in\left[H_{0}-\delta_{H}, H_{o}+\delta_{H}\right]
\end{gathered}
$$

where $p_{i j}$ is a pixel of the patch $S_{\text {patch }}$ and $H($.$) is the H-$ value (between $0^{\circ}$ and $360^{\circ}$ ) of the HSV channel for some pixel.

Considering some frame and the pixel set $S_{p l}$ corresponding to the pipeline representation, a patch of the line is, for instance, a ROI (Region of Interest) of the frame that partially covers the pipeline, i.e., $S_{\text {patch }}$ is a subset of $S_{p l}$. In general, $S_{\text {patch }}$ is an irregular set of connected pixels belonging to $S_{p l}$, including a set representing a segment of pixels. The parameter $\mathrm{H}_{0}$ is the mean $\mathrm{H}$-value of the patch given by the hue channel and the $\delta_{H}$ is the dispersion of that hue value.

One might argue that any subset $S_{i} \subset S_{p l}$ would has the same attribute. However, due to the nonuniformity of the luminance in the picture, this asseveration does not necessarily apply.

\section{B. Edge Predicate}

One of the usually employed attributes in subaquatic navigation is related to the shape of objects in water. Here we define a predicate of homogeneity for the 2D-pofile of the trackeable object (which is a detectable stretch of the pipeline) as

$$
P_{e}\left(S_{\text {line-stretch }}\right) \triangleq \text { true if for all } p_{i j} \in S_{\text {line-stretch }}
$$


With

$$
S_{\text {line-stretch }} \equiv S_{H}
$$

where $S_{\text {line-stretch }}$ is a pixel set which coincides with some $S_{H}$ that contains the pixels of an rectilinear segment detector or border detector. In this work, the Hough Transform for straight segments of the pipe stretch represented in the frame and the Canny edge detector were employed with this end. However, the choice of other detectors is totally feasible.

\section{Straight Segment Detection}

Hough transformation is a widespread way to detect a set of connected pixels that roughly satisfy an analytical equation, in our case a linear equation. The reason to employ the Hough Transform is that the line detection can be carried out taking into account uncertainties in different degrees. This means, one can identify not only a straight line of pixels but also a strip around the line. The width of the strip is related to the size of the uncertainty.

Certainly, employing a Hough parametric space, any shape can be written in parametric equation form (Antolovic, 2008). The detection is based on the pointline duality relation, which is a singularity-free alternative with respect to other parameterization forms. Computationally the line written in the form employs two parameters, namely $r$ and $\theta$,

$$
\begin{aligned}
& y=-\left(\frac{1}{\tan \theta}\right) x+\frac{r}{\sin \theta} \\
& r(\theta)=x_{i} \cos \theta+y_{i} \sin \theta
\end{aligned}
$$

where $r$ is the distance from the frame origin to the line and $\theta$ is the angle of the vector from the origin to this closest point. So the pair $(r, \theta)$ pertains to the line that crosses that closest point. All points $\left(x_{i}, y_{i}\right)$ in the space $\mathrm{x}$ $\mathrm{y}$ that belong to the line, are mapped in a unique point $\left(r_{0}, \theta_{0}\right)$ according to $(6)$

However, the accumulation of points around $\left(r_{0}, \theta_{0}\right)$ is an evidence that they all pertain to an extended strip representing the line. Fixing a ball of radius $\varepsilon_{0}$ around the mean value $\left(r_{0}, \theta_{0}\right)$, the width of a strip is determined.

Nevertheless, in noisy submarine scenes it is fully possible that there exist more than one cluster as object candidate, i.e., pairs $\left(r_{i}, \theta_{i}\right)$ in balls of radius $\varepsilon_{i}=\varepsilon_{0}$. Hence, one can implement an explicit voting procedure by counting the points inside every cluster. To this end many associated storage bins are created containing point coordinates and point counts (number of votes). A typical reasoning is that the most likely cluster representing the stripe of the pipeline is that bin which has the highest count.

The approach was significantly improved, above all in the performance of the voting scheme, producing a transform more robust to the detection of spurious lines (Fernandes and Oliveira, 2008).

\section{IV.LINE DETECTION}

Formally, if we assume that the storage bin count $b_{i}$ produces $b_{i}\left(p_{j}\right)=n_{i}$, it is $n_{i}$ votes from $n_{i}$ pixels $p_{j}$, thus

$$
b_{i}\left(p_{j}\right)=n_{i} \rightarrow S_{\text {hough }}=\left\{p_{j}\right\}
$$

where $\mathrm{N}$ is the number of bins, then will determine the most likely set, referred to as $S_{\text {line-stretch }}$, that represents the pipeline stretch in this frame. This is the line detector that will be applied in the optimal procedure as will be outlined in the next sections.

$$
\max _{i=1 \ldots N} b_{i}\left(p_{j}\right)=\max _{i=1 \ldots N} n_{i} \rightarrow S_{\text {hough }} \equiv S_{\text {line-stretch }}
$$

\section{V.VISION-BASED SENSOR}

Now, we can argue that a combination of color and shape attributes will provide a lot of information to detect the pipeline with a high success rate, including the cases in which environmental conditions of light are continuously changing.

According to the state of the art, a gray scale and a color model are the usual starting point to the primary objective of segmentation. There are many equivalent color models and also there exists the possibility to work in many color spaces simultaneously before fusing all local results with the technique $\mathrm{K}$-means clustering (Mignotte, 2008).

In this work we have mainly tested the HSV model due to the information of color is oriented to color properties rather than the advantages that other models (e.g., RGB, CMY, YIQ) possess in vision-oriented hardware.

Roughly speaking, we are of the opinion that the sensibility of each channel with respect to the luminance levels is significantly high. This observation means that by employing, for instance, multilevel thresholding, those parameters like mean value and length of the thresholds have to be selected carefully in a commissioning phase attending the range of changes of luminance and illuminance of the scene.

The basic algorithm is described here is more or less different to the technique that uses blob descriptors for peak detection with application to segmentation (Sattar and Dudek, 2006). Fig. 2 illustrates steps taken to achieve the stated objective.

The first step is the calibration phase that is accomplished by manually picking up a patch (ROI) of the camera frame over the silhouette of the pipeline. The color information contained in this patch gives way to generate thresholds, i.e., mean values and interval lengths of the thresholding process carried out for every channel of the HSV model. The interval lengths can be extended based on experience and knowledge, above all preventing the uncertainty that will be present in the luminance during the course of the application.

The algorithm begins with the patch segmentation. To this end, an optimal Canny edge detector is applied over a greyscale of the initial frame in order to establish as many real edges in the scene as possible. Here, the borders of the line would have to appear together with many edges of the scene. Additionally, color information is separated into channels for the hue $\mathrm{H}$, saturation $\mathrm{S}$, and value $\mathrm{V}$. Using the settings for the color thresholding, three binarized pictures (one for each channel) are originated. The most informative picture is accomplished when the 
Canny edge binarized result and the three binarized pictures of the color segmentation are combined in a pixel wise AND operation. Over this result, the Hough transform is applied according to Eqs. (7)-(8). In this way, the pattern of the pipeline stretch is detected as a maximization of the bin counts, given rise to the estimated values $\alpha$ and $x_{L}$.

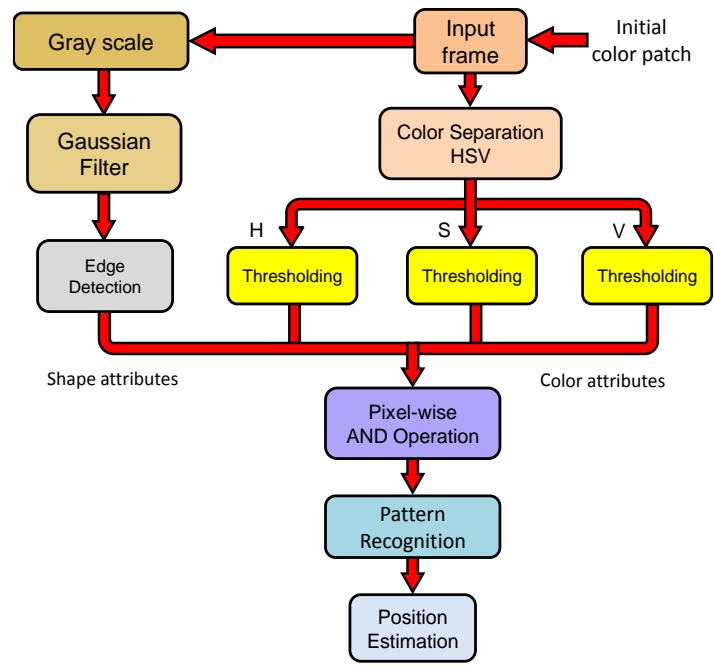

Figure 2. Vision-based sensor

\section{VI.ADAPTIVE VISION-BASED SENSOR}

We have mentioned the high sensibility of the color properties of the scene by changing luminance. Particularly the hue histogram tends to shrink and concentrate around a bluish tint. By marked darkening of the scene, color composition will give biased and partial information, masking any vestige of edge. When this extreme case exists, color information is irrelevant in front of the details provided by greyscale.

In order to reach stability in the ongoing identification of the pipe and take plenty advantage of color information, the basic technique described above has to be modified, for instance by equipping the algorithm with some adaptive mechanism.

The structure of the adaptive method we are presenting is composed of three elements, namely: a) the generator of estimation errors, b) the line parameter detector and estimator, and c) the adjust mechanism with the objective function.

\section{A. Generation of Estimation Errors}

In the navigation and guidance of unmanned underwater vehicles, the induced motions are smooth so that we can argue that the profile of the line in the frame changes smoothly. Usually, a vision-based controller saves the past estimated line parameters $\left(x_{L}, \alpha\right)$ for different purposes of control and monitoring issues. Moreover, the stretch of the pipeline that is visualized in the frame has commonly a smooth curvature. This means that the actual position of the line does not differ significantly from the previous position.

Accordingly, one can think up a trust zone described by a straight strip containing the last estimated line in the middle (Jordán et al., 2010). There are guidelines to select the width of the trust zone according to the processing frame rate and vehicle velocity, for instance, $5 \%$ of the frame height in pixel. Then, if the new estimated line lies in trust zone we will say that the estimation is reliable (see Fig. 3).

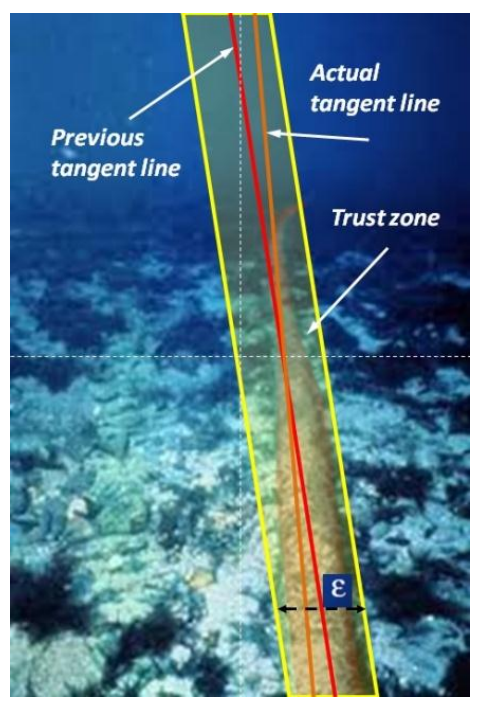

Figure 3. Trust zone of the sensor of width $\varepsilon$

In this sense, we postulate a binary error $\varepsilon_{p}$ for the result of the position estimation according to

$$
\text { If }\left\|\left(\alpha_{0}-\alpha_{1}\right),\left(x_{L_{0}}-x_{L_{1}}\right)\right\| \leq \delta_{p} \text { then } \varepsilon_{p}=1
$$

Otherwise $\varepsilon_{0}=0$, or if the estimation of $\left(\alpha_{0}, x_{L_{0}}\right)$ fails $\varepsilon_{p}=0$,

where $\left(\alpha_{0}, x_{L_{0}}\right)$ and $\left(\alpha_{1}, x_{L_{1}}\right)$ are the actual and past estimates, respectively, and $\delta_{p}$ is a real-valued number which depends in closed form on the strip width, for instance, $\delta_{p}=25$ corresponding to $\left|\alpha_{0}-\alpha_{1}\right| \leq 15^{\circ}$ and $\left|x_{L_{0}}-x_{L_{1}}\right| \leq 20$ pixels.

Another equivalent postulation of the performance error can be done according to the storage bin in the Hough Transform. One can define a trust zone in the space $(r, \theta)$ in the following way. Consider the balls for the storage bins defined for the success of the actual and the last estimation. Now, let these sets in the space $(r, \theta)$ be referred to as $B_{0}$ and $B_{1}$, respectively. Thus

$$
\text { if } B_{0} \cap B_{1} \neq \phi \text { then } \varepsilon_{p}=1
$$

Otherwise $\varepsilon_{p}=0$, or if the estimation of $\left(\mathrm{r}_{0}, \theta_{0}\right)$ fails $\varepsilon_{p}=0$,

Finally, the certainty of a successful line detection is given when $\varepsilon_{p}=1$.

\section{B. Line Parameter Detection and Estimator}

Equal to the basic algorithm, the line is detected by applying Eqs. (7)-(8). From this result a geometric segment is estimated through the $\alpha$ and $x_{L}$. To this end, a simple method like a minimal-squares-error technique can be applied.

\section{Adjust Mechanism with an Objective Function}

We know that the pixel wise AND operation over the shape-color segmented picture in the fixed-parameter 
algorithm will enhance the results if we compare it with that obtained through a gray-scale segmentation.

Taking the threshold magnitudes of the HSV channels as influential variables, we can specify the mean value $m_{i}$ and the interval length $\sigma_{i}$ to represent these magnitudes with i referring to the different channels as $\mathrm{H}, \mathrm{S}, \mathrm{V}$.

Now, supported by the fact that the trajectory of the estimations $\left(\alpha, x_{L}\right)$ are smooth we can state following conjecture in probabilistic terms. Let $P($.$) be the$ probability function defined on a random event of the estimation error. We state

$$
P_{\text {max }}=\max _{\max V} P\left(\varepsilon_{p}=1\right)
$$

where $P_{\max }$ is the maximal probability and $J$ is an objective function defined as

$$
\begin{gathered}
J=n_{\text {hough }}\left(m_{k}, \sigma_{k}\right) \text { with } k \in\{H, S, V\} \\
n_{\text {hough }}=b_{\text {hough }}\left\{p_{j}\right\} \text { and } S_{\text {hough }}=\left\{p_{j}\right\}
\end{gathered}
$$

Then, maximizing $\mathrm{V}$ in the space of the influential variables $m_{k}, \sigma_{k}$ with $k \in\{H, S, V\}$, will imply the maximization of the bin count of the Hough Transform output. It is.

$$
\begin{gathered}
\max _{m_{k}, \sigma_{k}, k \in\{H, S, V\}} J=b_{i}\left(p_{j}\right)=\max _{m_{k}, \sigma_{k}, k \in\{H, S, V\}} n_{i} \\
S_{i}=\left\{p_{j}\right\} \equiv S_{\text {line-stretch }}
\end{gathered}
$$

where $S_{\text {line-stretch }}=\left\{p_{j}\right\}$ contains the points $\left(r_{j}, \theta_{j}\right)$ from which the line is estimated by means of a meansquare-errors method.

Clearly, the parameter space of the influential variables has 6 dimensions. Since the maximization process has to be solved frame by frame, we can simplify the process by adjusting only the mean values for fixed interval lengths $\sigma_{k}$ specified previously.

The way the approach reach a maximum of $\mathbf{J}$ in the space $\left(m_{H}, m_{S}, m_{V}\right)$ is implemented by a gradient method. The adjust trajectories of $\left(m_{H}, m_{S}, m_{V}\right)$ will follow the path of maximal climbing, it is the direction of the gradient evaluated on the trajectory.

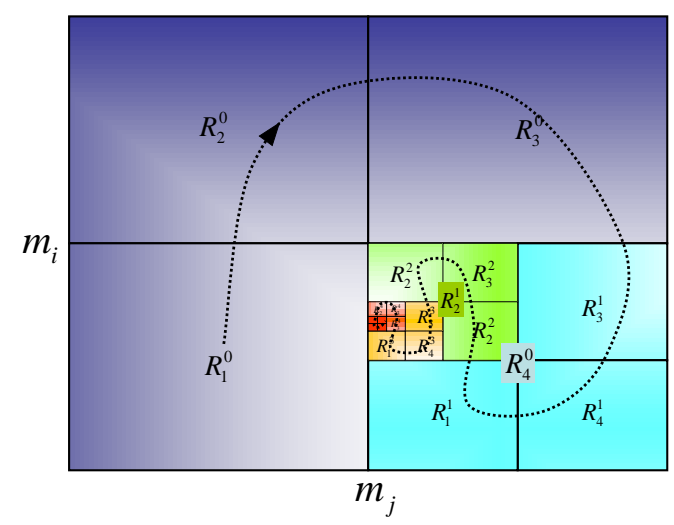

Figure 4. Adjust mechanism

In (Jordan and Bustamante, 2008) a method is developed, which is very appropriate for optimizations in high dimensional spaces. Briefly, it consists in segmenting progressively a compact region of the space of parameters (see Fig. 4 for 2 parameters) and evaluating
(14)-(15) in the geometric centers of every region $R_{j}^{i}$. When a minimal $\mathbf{J}$ is found in one region, one takes this region for a new, smaller segmentation. Here, one can employ a Fibonacci Series to define the new region size, for instance, size $\left(R_{j}^{i+1}\right)=\gamma \operatorname{size}\left(R_{j}^{i}\right)$ where $\gamma=1 / 2,1 / 3$, $1 / 5,1 / 8,1 / 13 \ldots$ and so on. The optimization process can be interrupt when the gradient satisfies

$$
\nabla J_{i+1}=J\left(R_{j}^{i}\right)-J\left(R_{j}^{i+1}\right) \leq \varepsilon
$$

where $\varepsilon$ is some small positive real value the optimization process can be finished.

Finally, the adaptive vision-based sensor is illustrated in Fig. 5.

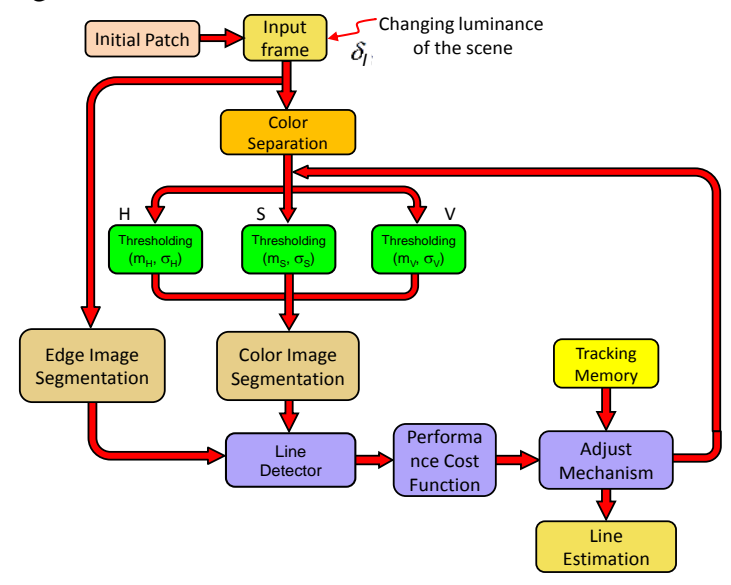

Figure 5. Adaptive vision-based algorithm

\section{EXAMPLE OF APPLICATION}

For the validation of the sensor performance, experimental setups have specifically been mounted. A colored line was deposit on the floor in a water tank. The scene was arranged in such a form that other objects like colored stones, mosses and additional colored lines with different hues are present. So the scene looks approximately like a natural scenario. Besides, the scene was externally illuminated with large variations of the intensity during the test. A subaquatic camera was conducted at a certain altitude along the line and the resulting videos were processed in the framework of the proposed approach. A commissioning phase consisting in picking up manually a patch of the line, served to extract initial color attributes.

Many examples are carried out under this setup employing lines of two colors, red and green. The results of all runs are very similar. To illustrate the performance of the approach, we described the identification case of a green line.

In order to compare the adaptive sensor performance, an auxiliary sensor with fixed (but good tuned) parameters is developed with such purpose. The settings for the adaptive sensor were: $\mathrm{H}=65 \pm 10, \mathrm{~S}=170 \pm 75$ and $\mathrm{V}=197 \pm 50$. These values are determined by taking manually an initial patch of the line in the picture. The adaptive algorithm performs the optimization on the $\mathrm{V}$ channel dominantly.

Fig. 6 shows the sensor evolution in three instants of the run. On the left of the figure, the original frames are 
depicted. On the right, the identified lines are superposed together with the original frames. Here, one can see three identified lines: the previously line (in yellow), the line provided by a fixed-parameter sensor (in green) and the line estimated by the adaptive sensor (in blue).

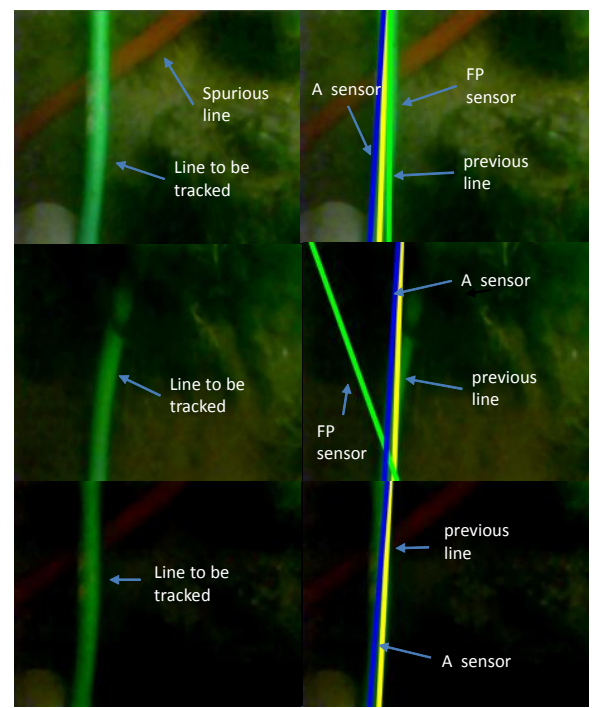

Figure 6. Identification of a green pipeline. Three instances of the video

While the adaptive sensor shows full success in all three instances, the fixed-parameter sensor exhibits a misestimation and a fail in the second and third instances. The cause for this bad performance is the low level of the illuminance in this instances (see Fig. 7).

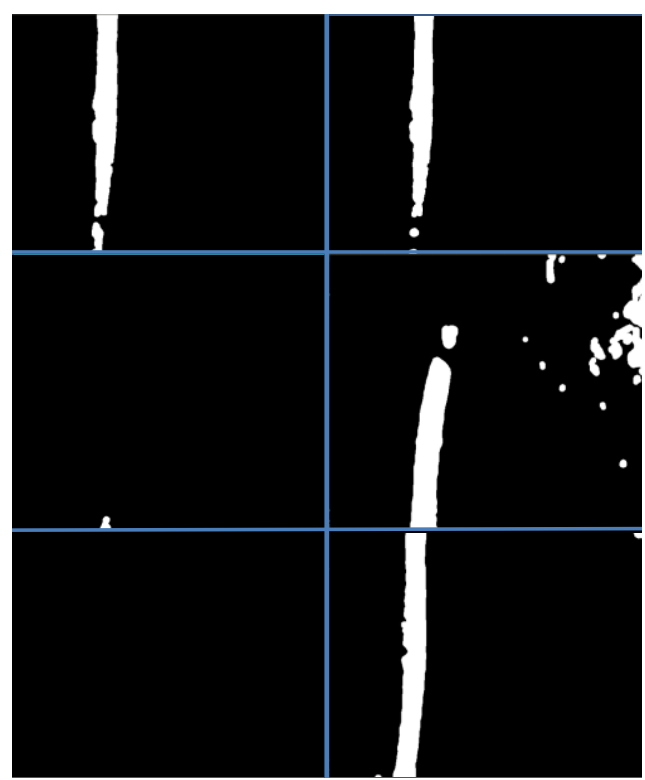

Figure 7. Detection of straight objects. Left: for the fixed-parameter sensor (FP). Right: for the adaptive sensor (A)

The next figures give out more explanation to the correlation between illumination level and sensor success. Fig. 8 shows the evolution of the two approaches comparatively during the experiment. One notes that affectation of poor illuminated scenes is high in the fixedparameter sensor, but it appears to be insignificant in the adaptive sensor. Additionally, the periods of low illumination are reflected from the mean value of the channel V displayed in see Fig. 9. There is a coincidence of the fails in the fixed-parameter sensor with the periods of low illumination.

The evolution of the storage bin counts in Fig. 10, clearly reveals the fails of the fixed-parameter sensor when the bin count is zero for long periods. Also here one can note the correspondence between these periods and those of low-level illumination.

\section{CONCLUSION}

In this work a vision-based sensor for identification of a pipeline in underwater environments is designed. The work emphasizes applications with low-level and changing luminance of the underwater scene and how these environmental conditions may affect the sensor performance. Thus a sensor with adaptive features is stated. It performs simultaneously shape and color image segmentation provided by four different sources, namely edge and color thresholdings, in which the color is separated according to an HSV model. To accomplish the adaptation, the thresholds on the three HSV channels are tuned. The adaptive technique employs a line identification error generator, an adjust mechanism and an objective functional over the threshold parameter space. An ad-hoc gradient method to maximize the objective functional is developed. In a comparison with fixed-parameter technique, our approach has proven to have a superior success rate in all illumination levels of the scene. An implementation with appropriate hardware to support the optimization in three or more parameters remains open for a future work.

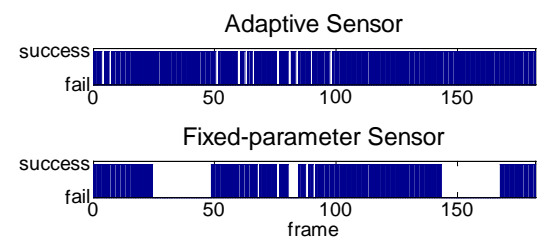

Figure 8. Tracking of the line in green. Evolution of the performance errors in the adaptive sensor (top) and the fixed-parameter sensor (bottom)

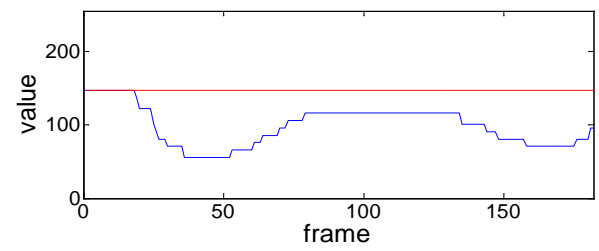

Figure 9. Evolution of the V-value in the adaptive sensor (in blue) an in the fixed-parameter sensor (in red)

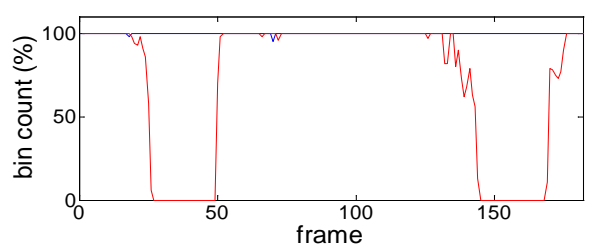

Figure 10. Evolution of maximal bin count in the adaptive sensor (in blue) and in the fixed-parameter sensor (in red) 


\section{REFERENCES}

[1] D. Antolovic, "Review of the Hough transform method, with an implementation of the fast Hough variant for line detection," Technical Report TR663, Indiana University, April 2008.

[2] H. D. Cheng, X. Jiang, A. Sun, and J. Wang, "Color image segmentation: Advances and prospects," Pattern Recognition 34, vol. 12, 2001.

[3] L. A. F. Fernandes and M. M. Oliveira, "Real-time line detection through an improved Hough transform voting scheme," Pattern Recognition, vol. 41, no. 1, pp. 299-314, January 2008.

[4] M. A. T. Figueiredo and A. K. Jain, "Simultaneous feature selection and clustering using mixture models," IEEE Transactions on Pattern Analysis and Machine Intelligence, vol. 26, no. 9, 2004.

[5] R. Harrabi and B. E. Ben, "Color image segmentation using automatic thresholding techniques," in Proc. SSD'2011, Tunisia 2011, 1-6.

[6] M. A. Jordán, C. E. Berger, J. L. Bustamante, S. Hansen, "Path tracking in underwater vehicle navigation-on-line evaluation of guidance path errors via vision sensor," in Proc. 49th IEEE Conf. on Decision and Control, Atlanta, USA.

[7] M. Jordan and J. Bustamante, "Auto-sintonización basada en la optimización de la performance en vehículos subacuáticos guiados adaptivamente," XXI Congreso Argentino de Control Automático, 2008.Law, M.H.C., Sept. 1-3.

[8] M. Mignotte, "Segmentation by fusion of histogram-based K means clusters in different color spaces," Image Processing, IEEE Trans., vol. 17-5, pp. 780-787, 2008.

[9] J. Sattar and G. Dudek, "On the performance of color tracking algorithms for underwater robots under varying lighting and visibility," in Proc. IEEE International Conference on Robotics and Automation ICRA, Orlando, Florida, May 2006.

[10] J. Sattar and G. Dudek, "Robust servo-control for underwater robots using banks of visual filters," in Proc. IEEE International
Conference on Robotics and Automation, Kobe, Japan, May 2009, pp. 3583-3588.

[11] L. G. Shapiro and G. C. Stockman, "Computer vision," New Jersey, Prentice-Hall, 2011, pp. 279-325.

[12] R. Szeliski, Computer Vision: Algorithms and Applications, Springer, Sep. 3, 2010.

Mario Jordan received the Dipl.-Ing. degree in Electro-Mechanical Engineering with orientation in Control Systems from the Universidad Nacional of San Juan, Argentina, in 1983, and the Ph.D in Control Systems from the Institute of Control at the Technical University of Darmstadt, Germany, in 1990. From 1992 to 1994, he held a fellowship of the Foundation for Industrial Research, Cologne, Germany, for pursuing postdoctoral work. He was Assistant Professor at the Institute of Control Systems, San Juan, Argentina, from 1992 to 1994, and Associated Professor at the National University of the South, Bahia Blanca, Arg., from 1995 to 2011. He is a Research Fellow of the Council for Scientific and Technological Research, Argentina, since 1995. He has authored more than 170 publications and participated in scientific books. He leads different technological projects and his current scientific research at the Argentinean Institute of Oceanography. His main interests are nonlinear and adaptive control theory, and systems for autonomous guidance and vision-based control of underwater vehicles

Emanuel Trabes was born in Buenos Aires, Arg. He received the Bachelor Engineering degree in Electronic Engineering from the Universidad Nacional de San Luis, San Luis, Arg. in 2013. He is currently pursuing his $\mathrm{PhD}$ degree in system control from the Universidad Nacionaldel Sur, Arg., funded by the Council for Scientific and Technological Research. His research interest includes control of underwater vehicles, image processing and FPGA-based design. 